\title{
Oropharyngeal Dysphagia in Acute Stroke Patients
}

\author{
Faezeh Asadollahpour ${ }^{1,}$; Kowsar Baghban $^{2}$; Mojgan Asadi ${ }^{3}$; Ehsan Naderifar ${ }^{1}$; Maryam \\ Dehghani $^{1}$
}

${ }^{1}$ Department of Speech Therapy, Health Promotion Research Center, Zahedan University of Medical Sciences, Zahedan, IR Iran

2 Department of Speech Therapy, Hamadan University of Medical Sciences, Hamadan, IR Iran

${ }^{3}$ Department of Speech Therapy, Semnan University of Medical Sciences, Semnan, IR Iran

${ }^{*}$ Corresponding author: Faezeh Asadollahpour, Department of Speech Therapy, Health Promotion Research Center, Zahedan University of Medical Sciences, Zahedan, IR Iran. E-mail: faezehasadollahpour@yahoo.com

Received: June 29, 2014; Accepted: August 5, 2014

Background: The objective of this study was to investigate the prevalence of different kinds of swallowing disorder and it's severity in patients after stroke.

Patients and Methods: In this cross sectional study, 91 consecutive stroke patients were screened by the Northwestern Dysphagia Patient Check Sheet (NDPCS) and the Gugging Swallowing Screen (GUSS).

Results: Forty seven percent of those assessed demonstrated signs of oropharyngeal dysphagia. Mild dysphagia was seen in (10.98\%) patients, moderate dysphagia was present in $14.28 \%$ and $22 \%$ patients had severe dysphagia.

Conclusions: Dysphagia is common after acute stroke and its presence can have serious consequences. These results emphasize the importance of assessment and management of swallowing function in stroke patients.

Keywords: Stroke; Dysphagia; Oropharyngeal

\section{Introduction}

Dysphagia is a term derived from the Greek words dys (difficulty) and phagia (to eat) [1]. It is a symptom which indicates a delay in the passage of solids or liquids from the oral cavity to the stomach $[1,2]$. Dysphagia is a serious consequence of stroke [3], because of the risk of aspiration, malnutrition, dehydration, long-term hospitalization, airway obstruction, and ultimately death [4]. Dysphagia and related complications are associated with increased mortality, comorbidity and increased health care costs [5]. However, there is emerging evidence that early detection of dysphagia in patients with stroke reduces not only these complications but also reduces length of hospital stay and overall healthcare expenditures [6].

A number of tests for evaluating dysphagia have been developed. Northwestern Dysphagia Patient Check Sheet (NDPCS) and Gugging Swallowing Screen (GUSS) are two of these screening tests. According NDPCS identified patients with pharyngeal stage disorder, aspiration, oral stage disorders, and pharyngeal delay. GUSS described both the severity of dysphagia and the risk of aspiration. Investigate of the prevalence of dysphagia and related complications in stroke patients will be critical to guide the design of future research aiming to assess benefits of dysphagia interventions [7]. Therefore, this study aimed to determine the prevalence of swallowing disorders and its types and severity in Iranian acute stroke population, and to study possible associations between clinical and demographic characteristics of patients and presence of dysphagia.

\section{Patients and Methods}

For this cross sectional study, 91 patients with acute stroke were recruited (sample size was calculated using the formula). Sequential patients admitted to the inpatient stroke unit at hospitals in Zahedan between May 2013 and August 2013, who met the following inclusion criteria were recruited as the sample for this study: stroke identified by neurological examination and no prior history of oropharyngeal dysphagia by patient and care giver report. We also included those patients who had no previous head and neck surgery or trauma that may impact swallowing ability. Exclusion criteria were multiple infarcts visible on computed tomography or MRI and coma (patients with severe decrease level of consciousness were excluded).

All patients were assessed by the neurologist to establish the diagnosis of stroke. Stroke was confirmed by documentation of acute infarct by either CT or MRI in the patients studied. Furthermore, informed consent obtained from either the patient or the family. The study consisted

Copyright (C) 2015, Zahedan University of Medical Sciences. This is an open-access article distributed under the terms of the Creative Commons Attribution-NonCommercial 4.0 International License (http://creativecommons.org/licenses/by-nc/4.0/) which permits copy and redistribute the material just in noncommercial usages, provided the original work is properly cited. 
of collecting demographic and clinical data relating to patient identification.

The evaluation of swallowing was performed with the NDPCS and GUSS (these screening tools are valuable and reliable for detecting dysphagia). NDPCS is a screening test includes 28 items divided in to 5 categories. This test has 4 items in medical history variables categories, 6 items in behavioral variable, 2 items in gross motor, 9 items in oromotor test, and 7 observations during trial swallows. Each item of these categories is scored as "safe" or "unsafe." During trial swallows, patients were requested to drink $1 \mathrm{~mL}$ thin liquid, $1 \mathrm{~mL}$ pudding and a quarter of a Lorna Doone cookie (if chewing was possible) [8] and were observed during swallowing. According to the results of this test, patients who scored as "unsafe" on at least two out of the three variables (number of variables: 24-26-1), were defined as having aspiration. If a patient was scored as "unsafe" on the variable of "dysartheria" in NPDCS, oral stage swallowing difficulty was identified. The patients who scored as "unsafe" on at least two of three variables (number of variables: 16 - 25 and to be unsafe on more than 8 of the 28 items), pharyngeal delay was identified. At last, dysphagia was defined as having at least one of these 4 kinds of disorders. The GUSS offers a quick and reliable method to identify stroke patients with dysphagia and aspiration risk [9]. The GUSS consists of an indirect swallowing test and 3 direct swallowing tests. The indirect swallowing test is a simple saliva swallow, while in the direct swallowing tests patients swallows semisolid, liquid and solid textures. Furthermore, the GUSS scores yielded 4 categories of severity, zero to 9 points are rated severe, 10 to 14 points moderate, 15 - 19 points mild, and 20 points as no dysphagia. All patients underwent evaluation of the swallowing with NDPCS and GUSS. Finally, data analyses were performed using SPSS-11.
For statistical analysis of demographic variables between dysphagic and non-dysphagic patients, we used an independent-sample t-test. To identify possible associations between clinical and demographic features of patients and the presence of dysphagia, statistic tests were carried out, taking the presence of dysphagia as the dependent variable and other independent variables (independent variables: sex, age, duration of hospitalization).

\section{Results}

In this study participated 52 men (57\%) and 39 women (43\%), with an average age of $60 \pm 10.2$ years, ranging from 25 to 89 years. Dysphagia was most prevalent among the patients aged 64.5 years and over. In patients with dysphagia, the mean length of hospital stay was prolonged to 40.6 days (30 to 61 days) compared with 21.5 days (15 to 31 days) for those with a normal swallow $(\mathrm{P} \leq 0.01$ ) (Table 1). Thus, dysphagic patients had significantly longer duration of hospital stay compared to non-dysphagic patients.

The frequency of dysphagia was not significantly different in term of baseline characteristics such as age, sex and stroke severity (NIHSS). Swallowing performance assessment showed that $43(47 \%)$ stroke patients had dysphagia and 48 (53\%) patients were normal in this task. Among those patients with swallowing disorders, 55 (60.43) had pharyngeal stage disorder, 49 (53.85\%) oral stage swallowing abnormalities, 66 (72.52\%) pharyngeal delay and 63 (69.23\%) aspiration. Pharyngeal delay stage disorders were the most common observed impairment (Table 2). According to GUSS, in the group of patients with dysphagia, mild dysphagia was seen in 10 (10.98\%) patients, moderate dysphagia was present in $13(14.28 \%)$ and 20 (22\%) patients had severe dysphagia. In direct swallowing test, swallowing of semisolid texture was better than liquids and swallowing of liquids was better than solid.

Table 1. Demographic and Clinical Features of Patients

\begin{tabular}{lccc}
\hline Variables & Dysphagic & Non Dysphagic & P Value $^{\mathrm{a}}$ \\
\hline Age (mean), $\mathbf{y}$ & 64.5 & 60.2 & 0.001 \\
Gender & & & $>0.05$ \\
$\quad$ Male & 23 & 29 & \\
Female & 20 & 19 & 0.001 \\
\hline Duration of hospital stay, days & $40.6(30-61)$ & $21.5(15-31)$ & \\
\hline
\end{tabular}

a Descriptive statistical analysis and t-test.

Table 2. Swallowing Assessment in Dysphagic and Non-Dysphagic Groups ${ }^{\text {a }}$

\begin{tabular}{lccc}
\hline Screening Tool & Stage & Dysphagic & Non Dysphagic \\
\hline NDPCS & Pharyngeal delay & 72.52 & 27.48 \\
& Aspiration & 69.23 & 30.77 \\
& Oral stage disorder & 53.85 & 46.15 \\
\hline
\end{tabular}

\footnotetext{
a Data are presented as \%.
} 


\section{Discussion}

The finding of this study showed important results. First, swallowing disorder occurred in approximately $47 \%$ of the stroke patients. This prevalence rate falls within the range reported from previous studies. According to these studies, the prevalence of dysphagia in stroke patients was 17 - 81\% depending on the timing, methods, and criteria of diagnosis $[10,11]$. Despite the frequency and potential seriousness of dysphagia in stroke patients in Iran, they are often not sought systematically in patients admitted to hospital with stroke and few of them received a swallowing treatment. In this study, $60.43 \%$ of patients had pharyngeal stage disorder, $53.85 \%$ oral stage disorder, $72.52 \%$ pharyngeal delay, and $69.23 \%$ aspiration. Pharyngeal delay stage disorders were the most common observed impairment (72.52\%). This finding agrees with the studies from Logemann et al. [8]. Our study demonstrated that dysphagia was related to duration of hospital stay and its duration increased with severe dysphagia. These finding are similar to the finding of others [12-14]. A longer length of stay has significant cost implications for the hospital as well as possible adverse health outcomes for the patients. We have shown that stroke patients are better at swallowing semisolid textures than liquids and solid. Thus, it is essential to recommend semisolid swallowing before liquid and solid swallowing to reduce the risk of aspiration. This finding is also in line with those of Trapl et al. [9] and Clave et al. [15] and reflects the significance of textures modification. Therefore, liquids should not be one of the first offered items, because it is considered as the least safe consistency for deglutition. In our study, we described both the severity of dysphagia and the risk of aspiration. According to our study, severe dysphagia is common in stroke patients. This finding is in line with those of Trapl et al. [9]. Limitation of this study was the small size and the lack of longitudinal data. Longitudinal studies with a large population are guaranteed to confirm these results and to determine whether dysphagia persist in some individuals with stroke. Future studies should investigate the impact of different kinds of stroke on long term swallowing ability and nutritional recommendations. In conclusion, dysphagia is a common clinical presentation following stroke with potentially devastating effects on the individual physical and psychological well-being. Identification of dysphagia reduced the risk of developing pneumonia, duration of hospital stay and etc. In this study, dysphagia identification was performed using clinical screening tools GUSS and NDPCS. Because of the high prevalence of dysphagia in stroke patients, our finding emphasized the impor- tance of assessment and management of swallowing in these patients.

\section{Acknowledgements}

The authors gratefully acknowledge the staff of speech therapy, department of speech therapy for their assistance in data collection for this study. This work was supported by a grant from the Zahedan University of Medical Sciences (Number: 6901 by Faezeh Asadollahpour).

\section{Funding/Support}

This study was supported by Zahedan University of Medical Sciences, Zahedan, IR Iran.

\section{References}

1. Manikantan K, Khode S, Sayed SI, Roe J, Nutting CM, Rhys-Evans P, et al. Dysphagia in head and neck cancer. Cancer Treat Rev. 2009;35(8):724-32.

2. Singh S, Hamdy S. Dysphagia in stroke patients. Postgrad Med J. 2006;82(968):383-91.

3. Martino R, Martin RE, Black S. Dysphagia after stroke and its management. CMAJ. 2012;184(10):1127-8.

4. Sorensen RT, Rasmussen RS, Overgaard K, Lerche A, Johansen AM Lindhardt T. Dysphagia screening and intensified oral hygiene reduce pneumonia after stroke. J Neurosci Nurs.2013;45(3):139-46.

5. Smithard DG, O'Neill PA, England RE, Park CL, Wyatt R, Martin DF, et al. The natural history of dysphagia following a stroke. Dysphagia.1997;12(4):188-93.

6. Martino R, Pron G, Diamant N. Screening for oropharyngeal dysphagia in stroke: insufficient evidence for guidelines. Dysphagia. 2000;15(1):19-30.

7. Martino R, Foley N, Bhogal S, Diamant N, Speechley M, Teasell R. Dysphagia after stroke: incidence, diagnosis, and pulmonary complications. Stroke. 2005;36(12):2756-63.

8. Logemann JA, Veis S, Colangelo L. A screening procedure for oropharyngeal dysphagia. Dysphagia.1999;14(1):44-51.

9. Trapl M, Enderle P, Nowotny M, Teuschl Y, Matz K, Dachenhausen A, et al. Dysphagia bedside screening for acute-stroke patients: the Gugging Swallowing Screen. Stroke. 2007;38(11):2948-52.

10. Meng NH, Wang TG, Lien IN. Dysphagia in patients with brainstem stroke: incidence and outcome. Am J Phys Med Rehabil. 2000;79(2):170-5

11. Suntrup S, Warnecke T, Kemmling A, Teismann IK, Hamacher C Oelenberg S, et al. Dysphagia in patients with acute striatocapsular hemorrhage. J Neurol. 2012;259(1):93-9.

12. Smithard DG, O'Neill PA, Parks C, Morris J. Complications and outcome after acute stroke. Does dysphagia matter? Stroke. 1996;27(7):1200-4.

13. Axelsson K, Asplund K, Norberg A, Eriksson S. Eating problems and nutritional status during hospital stay of patients with severe stroke. J Am Diet Assoc. 1989;89(8):1092-6.

14. Barer DH. The natural history and functional consequences of dysphagia after hemispheric stroke. J Neurol Neurosurg Psychiatry. 1989;52(2):236-41.

15. Clave P, Arreola V, Romea M, Medina L, Palomera E, Serra-Prat M. Accuracy of the volume-viscosity swallow test for clinical screening of oropharyngeal dysphagia and aspiration. Clin Nutr. 2008;27(6):806-15. 